\title{
Class of Trial Agent
}

National Cancer Institute

\section{Source}

National Cancer Institute. Class of Trial Agent. NCI Thesaurus. Code C90372.

A classification of the type of trial agent. 